UT-702,1995

NUS-HEP-95-03

\title{
Q-Deformed Oscillator Algebra and an Index Theorem for the Photon Phase Operator
}

\author{
Kazuo Fujikawa 円 \\ Department of Physics, University of Tokyo, \\ Bunkyo-ku, Tokyo 113, Japan \\ L. C. Kwek [2, and C. H. Oh 3 \\ Department of Physics, Faculty of Science, \\ National University of Singapore, Lower Kent Ridge, \\ Singapore 0511, Republic of Singapore.
}

\begin{abstract}
The quantum deformation of the oscillator algebra and its implications on the phase operator are studied from a view point of an index theorem by using an explicit matrix representation. For a positive deformation parameter $q$ or $q=\exp (2 \pi i \theta)$ with an irrational $\theta$, one obtains an index condition dim $\operatorname{ker} a-\operatorname{dim} \operatorname{ker} a^{\dagger}=1$ which allows only a non-hermitian phase operator with $\operatorname{dim} \operatorname{ker} \mathrm{e}^{i \varphi}-\operatorname{dim} \operatorname{ker}\left(\mathrm{e}^{i \varphi}\right)^{\dagger}=1$. For $q=\exp (2 \pi i \theta)$ with a rational $\theta$, one formally obtains the singular situation $\operatorname{dim} \operatorname{ker} a=\infty$ and $\operatorname{dim} \operatorname{ker} a^{\dagger}=\infty$, which allows a hermitian phase operator with $\operatorname{dim} \operatorname{ker} \mathrm{e}^{i \Phi}-\operatorname{dim} \operatorname{ker}\left(\mathrm{e}^{i \Phi}\right)^{\dagger}=0$ as well as the non-hermitian one with $\operatorname{dim} \operatorname{ker} \mathrm{e}^{i \varphi}-\operatorname{dim} \operatorname{ker}\left(\mathrm{e}^{i \varphi}\right)^{\dagger}=1$. Implications of this interpretation of the quantum deformation are discussed. We also show how to overcome the problem of negative norm for $q=\exp (2 \pi i \theta)$.
\end{abstract}

(To be published in Modern Physics Letters A)

\footnotetext{
${ }^{1}$ E-mail address: fujikawa@danjuro.phys.s.u-tokyo.ac.jp

${ }^{2}$ E-mail address: scip3057@nus.sg

${ }^{3}$ E-mail address: phyohch@nus.sg
} 


\section{Introduction}

The presence or absence of a hermitian phase operator for the photon is an old and interesting problem [1, 2, 3], see ref [4] for earlier works on the subject. Recently, one of the present authors [5] introduced the notion of index into the analysis of the phase operator. The basic observation is that the creation and annihilation operators of the oscillator algebra

$$
\left[a, a^{\dagger}\right]=1
$$

satisfy the index condition

$$
\operatorname{dim} \text { ker } a-\operatorname{dim} \text { ker } a^{\dagger}=1
$$

as seen from the conventional representation

$$
a=|0><1|+|1><2| \sqrt{2}+|2><3| \sqrt{3}+\cdots .
$$

The state vectors $\mid k>$ are defined by $N|k>=k| k>$, where $N$ is the number operator. The phase operator defined by [2]

$$
\begin{aligned}
\mathrm{e}^{i \varphi} & =\frac{1}{\sqrt{N+1}} a \\
& =|0><1|+|1><2|+|2><3|+\cdots
\end{aligned}
$$

faithfully reflects the index relation (2)

$$
\operatorname{dim} \operatorname{ker} \mathrm{e}^{i \varphi}-\operatorname{dim} \operatorname{ker}\left(\mathrm{e}^{i \varphi}\right)^{\dagger}=1 \text {. }
$$

On the other hand, if one assumes a polar decomposition $a=U(\phi) H$ with a unitary $U(\phi)$ and a hermitian $H$, one inevitably has

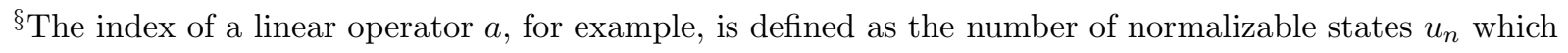
satisfy $a u_{n}=0$. 


$$
\operatorname{dim} \text { ker } a-\operatorname{dim} \text { ker } a^{\dagger}=0
$$

since the action of the unitary operator $U(\phi)$ is simply to re-label the names of the basis vectors. From these considerations, one concludes that the phase operator $\varphi$ in (团) cannot be hermitian, i.e., $e^{i \varphi}$ is not unitary. A truncation of the representation space of $a$ to $(s+1) \times(s+1)$ dimensions, however, generally leads to the index relation (6]) [5], and thus an associated phase operator $\phi$ could be hermitian. In fact, a hermitian phase operator $\phi$ may be defined by [3]

$$
\mathrm{e}^{i \phi}=|0><1|+|1><2|+|2><3|+\cdots+|s-1><s|+\mathrm{e}^{i \phi_{0}}|s><0|
$$

with a positive integer $s$ ( a cut-off parameter) and an arbitrary constant $\phi_{0}$. The unitary operator $\mathrm{e}^{i \phi}$ naturally satisfies the index condition

$$
\operatorname{dim} \operatorname{ker} \mathrm{e}^{i \phi}-\operatorname{dim} \operatorname{ker}\left(\mathrm{e}^{i \phi}\right)^{\dagger}=0,
$$

and gives rise to a truncated operator

$$
\begin{aligned}
a_{s} & =\mathrm{e}^{i \phi} \sqrt{N} \\
& =|0><1|+|1><2| \sqrt{2}+|2><3| \sqrt{3}+\cdots|s-1><s| \sqrt{s}
\end{aligned}
$$

with

$$
\operatorname{dim} \text { ker } a_{s}-\operatorname{dim} \text { ker } a_{s}^{\dagger}=0
$$

since $\quad a_{s}^{\dagger} \mid s>=0$.

The index relations (5) and (\$) clearly show the unitary inequivalence of $\mathrm{e}^{i \varphi}$ and $\mathrm{e}^{i \phi}$ even for arbitrarily large $s$. Since the kernel of $a_{s}^{\dagger}$ is given by $\operatorname{ker} a_{s}^{\dagger}=\{|s\rangle\}$ in (10), which is ill-defined in the limit $s \rightarrow \infty$, we analyze the behavior of $e^{i \phi}$ for sufficiently large but finite $s$. To make this statement of large $s$ meaningful, we need to introduce a typical number to 
characterize a physical system, relative to which the number $s$ may be chosen much larger. We thus expand a physical state as

$$
\left|p>=\sum_{n=0}^{\infty} p_{n}\right| n>
$$

The finiteness of $<p\left|N^{2}\right| p>$ requires

$$
\sum_{n} n^{2}\left|p_{n}\right|^{2}=N_{p}^{2}<\infty
$$

in addition to the usual condition of a vector in a Hilbert space,

$$
\sum_{n}\left|p_{n}\right|^{2}<\infty
$$

The number $N_{p}$ in (12) specifies a typical number associated to a given physical system $|p\rangle$. By choosing the parameter $s$ at $s>>N_{p}$, one may analyze the physical implications of the state $|s\rangle$, which is responsible for the index in (10), on the physically observable processes. It was shown in [5] that the origin of the index mismatch between (4) and (7), namely the state $|s\rangle$ in (7), is also responsible for the absence of minimum uncertainty states for the hermitian operator $\phi$ in the characteristically quantum domain with small average photon numbers.

A major advantage of the notion of index is that it is invariant under unitary time developments which include a fundamental phenomenon such as squeezing. Another advantage of the index idea lies in suggesting a close analogy between the problem of quantum phase operator with a non-trivial index as in (5) and chiral anomaly in gauge theory, which is related to the Atiyah-Singer index theorem. This was emphasized in Ref [5]. From an anomaly view point, it is not surprising to have an anomalous identity

$$
C(\varphi)^{2}+S(\varphi)^{2}=1-\frac{1}{2}|0><0|
$$

and an anomalous commutator 


$$
[C(\varphi), S(\varphi)]=\frac{1}{2 i}|0><0|
$$

for the modified cosine and sine operators defined in terms of $\mathrm{e}^{i \varphi}$ in (4) [2]

$$
\begin{aligned}
C(\varphi) & \equiv \frac{1}{2}\left\{\mathrm{e}^{i \varphi}+\left(\mathrm{e}^{i \varphi}\right)^{\dagger}\right\}, \\
S(\varphi) & \equiv \frac{1}{2 i}\left\{\mathrm{e}^{i \varphi}-\left(\mathrm{e}^{i \varphi}\right)^{\dagger}\right\}
\end{aligned}
$$

The notion of index is also expected to be invariant under a continuous deformation such as the quantum deformation of the oscillator algebra as long as the norm of the Hilbert space is kept positive definite.

\section{Q-deformation}

The purpose of the present note is to analyze in detail the behavior of the index relation under the quantum deformation of the oscillator algebra [6, 7]:

$$
\begin{aligned}
{\left[a, a^{\dagger}\right] } & =[N+1]-[N] \\
{\left[N, a^{\dagger}\right] } & =a^{\dagger} \\
{[N, a] } & =-a
\end{aligned}
$$

where

$$
[N] \equiv \frac{q^{N}-q^{-N}}{q-q^{-1}} .
$$

The parameter $q$ stands for the deformation parameter, and one recovers the conventional algebra in the limit $q \rightarrow 1$. The quantum deformation (17) is known to satisfy the Hopf structure [8, 9]. The algebra([17) accomodates a Casimir operator defined by [9]

$$
c=a^{\dagger} a-[N]
$$


which plays an important role in the following.

For a real positive $q$, we may adopt the conventional Fock state representation of the algebra (17) defined by [6, [7]:

$$
\begin{aligned}
c \mid 0> & =0 \\
a \mid 0> & =0 \\
<0 \mid 0> & =1 \\
N \mid k> & =k \mid k> \\
\mid k> & =\frac{1}{\sqrt{[k] !}\left(a^{\dagger}\right)^{k} \mid 0>} \\
a \mid k> & =\sqrt{[k]\left|k-1>, \quad a^{\dagger}\right| k>=\sqrt{[k+1]} \mid k+1>,}
\end{aligned}
$$

Here we have abbreviated $\mid k>_{q}$ by $\mid k>$. For a positive $q$, one thus obtains a representation

$$
a=|0><1| \sqrt{[1]}+|1><2| \sqrt{[2]}+|2><3| \sqrt{[3]}+\cdots
$$

which satisfies the index condition (2). The phase operator $\mathrm{e}^{i \varphi}$ is defined by [10]

$$
\begin{aligned}
\mathrm{e}^{i \varphi} & =\frac{1}{\sqrt{[N+1]}} a \\
& =|0><1|+|1><2|+|2><3|+\cdots
\end{aligned}
$$

so that the relation $a=\mathrm{e}^{i \varphi} \sqrt{N}$ holds. Evidently, expression (22) has the same form as that of Susskind and Glogower in [Q] , namely not only the index but also the explicit form of $\mathrm{e}^{i \varphi}$ itself remains invariant under quantum deformation.

If one extends the range of the deformation parameter $q$ to complex numbers, which is consistent only for $|q|=1$, one finds more interesting possibility. For previous discussions of this case from a finite dimensional cyclic representation, see papers in [11]. 
For a complex $q=\exp (2 \pi i \theta)$ with a real $\theta$, we adopt the following explicit matrix representation [12] of the algebra (17)

$$
\begin{aligned}
a & =\sum_{k=1}^{\infty} \sqrt{\left[k-n_{0}\right]+\left[n_{0}\right]}|k-1><k| \\
a^{\dagger} & =\sum_{k=1}^{\infty} \sqrt{\left[k+1-n_{0}\right]+\left[n_{0}\right]}|k+1><k| \\
N & =\sum_{k=0}^{\infty}\left(k-n_{0}\right)|k><k| \\
c & =\left[n_{0}\right]=\frac{1}{|\sin 2 \pi \theta|}
\end{aligned}
$$

Here the ket states $\mid k>$ stand for column vectors

$$
\left|0>=\left(\begin{array}{c}
1 \\
0 \\
0 \\
\vdots \\
\vdots
\end{array}\right),\right| 1>=\left(\begin{array}{c}
0 \\
1 \\
0 \\
\vdots \\
\vdots
\end{array}\right), \mid 2>=\left(\begin{array}{c}
0 \\
0 \\
1 \\
\vdots \\
\vdots
\end{array}\right), \ldots
$$

and the bra states stand for row vectors. The representation (20) may also be included in this matrix representation by letting $n_{0}=0$ and $c=0$. In eq(23) the Casimir operator $c$ for the algebra (17) is chosen so that $a^{\dagger} a>0$ and the absence of negative norm is ensured. We note that

$$
\begin{aligned}
{\left[k-n_{0}\right] } & =\frac{\sin 2 \pi\left(k-n_{0}\right) \theta}{\sin 2 \pi \theta} \\
& =-\frac{\cos (2 \pi k \theta)}{|\sin 2 \pi \theta|} \\
& \leq \frac{1}{|\sin 2 \pi \theta|}
\end{aligned}
$$


if one chooses $n_{0}$ as in (23),

$$
\left[n_{0}\right]=\frac{\sin \left(2 \pi n_{0} \theta\right)}{|\sin 2 \pi \theta|}=\frac{1}{|\sin 2 \pi \theta|}
$$

The argument of the square root in (23) is thus non-negative. This means that we have managed to overcome the problem of negative norm for $q=\exp (2 \pi i \theta)$. For irrational $\theta$

$$
\left[k-n_{0}\right]+\left[n_{0}\right]=0
$$

only if $k=0$.

We thus have the kernels, ker $a=\{\mid 0>\}$ and ker $a^{\dagger}=$ empty, and the index condition

$$
\operatorname{dim} \text { ker } a-\operatorname{dim} \text { ker } a^{\dagger}=1
$$

for a positive $q$ or $q=\exp (2 \pi i \theta)$ with an irrational $\theta$ : this index relation allows only the non-hermitian phase operator defined in (22), namely

$$
\begin{aligned}
\mathrm{e}^{i \varphi} & =\frac{1}{\sqrt{[N+1]+\left[n_{0}\right]}} a \\
& =|0><1|+|1><2|+|2><3|+\cdots
\end{aligned}
$$

This expression together with $[N+1]+\left[n_{0}\right] \neq 0$ shows that $\mathrm{e}^{i \varphi}$ and $a$ carry the same index, namely a unit index.

We next examine the representation (23) for a rational $\theta$. To be specific, we consider the case $q=\exp \left(\frac{2 \pi i}{(s+1)}\right)$, i.e., $\theta=\frac{1}{s+1}$ with a positive integer $s$ greater than one. One then obtains

$$
[s+1]=\frac{q^{s+1}-q^{-s-1}}{q-q^{-1}}=0
$$

In this case, the representation (23) becomes 


$$
\begin{aligned}
a= & \sqrt{\left[1-n_{0}\right]+\left[n_{0}\right]}|0><1|+\cdots+\sqrt{\left[s-n_{0}\right]+\left[n_{0}\right]}|s-1><s| \\
& +\sqrt{\left[1-n_{0}\right]+\left[n_{0}\right]}|s+1><s+2|+\cdots+\sqrt{\left[s-n_{0}\right]+\left[n_{0}\right]}|2 s><2 s+1| \\
& +\cdots \\
N= & \left(-n_{0}\right)|0><0|+\left(1-n_{0}\right)|1><1|+\cdots+\left(s-n_{0}\right)|s><s| \\
& +\left(s+1-n_{0}\right)|s+1><s+1|+\cdots+\left(2 s+1-n_{0}\right)|2 s+1><2+1| \\
& +\cdots \\
c= & {\left[n_{0}\right]=\frac{\sin \left(\frac{2 \pi n_{0}}{s+1}\right)}{\sin \left(\frac{2 \pi}{s+1}\right)}=\frac{1}{\sin \left(\frac{2 \pi}{s+1}\right)} }
\end{aligned}
$$

where $a^{\dagger}$ is given by the hermitian conjugate of $a$ and one may choose $n_{0}=\frac{s+1}{4}$.

One may look at the representation (31) from two different view points. One way is to regard it reducible into an infinite set of irreducible $(s+1)$ - dimensional representation specified by the eigenvalue of the Casimir operator $c=\left[n_{l}\right]\left(=\left[n_{0}\right]\right)$ where

$$
\begin{aligned}
n_{l} & =n_{0}-l(s+1) \\
& =\frac{1}{4}(s+1)-l(s+1)
\end{aligned}
$$

with $l=0,1,2, \cdots$. We note that $-n_{l}$ stands for the lowest eigenvalue of $N$. In this case, the basic Weyl block is given by

$$
\begin{aligned}
a_{s} & =\sqrt{\left[1-n_{0}\right]+\left[n_{0}\right]}|0><1|+\cdots+\sqrt{\left[s-n_{0}\right]+\left[n_{0}\right]}|s-1><s| \\
a_{s}^{\dagger} & =\left(a_{s}\right)^{\dagger} \\
N_{s} & =\left(-n_{0}\right)|0><0|+\left(1-n_{0}\right)|1><1|+\cdots+\left(s-n_{0}\right)|s><s| \\
c & =\left[n_{0}\right]=\frac{1}{\sin \left(\frac{2 \pi}{s+1}\right)}
\end{aligned}
$$


and other sectors are obtained by using the Casimir operator $c=\left[n_{l}\right]\left(=\left[n_{0}\right]\right)$ with the lowest eigenvalue of $N$ at $-n_{l}, l=1,2, \cdots$. This is the standard representation commonly adopted for the case $\theta=\frac{1}{(s+1)}$. This finite dimensional representation inevitably leads to the index condition[5]

$$
\operatorname{dim} \text { ker } a_{s}-\operatorname{dim} \text { ker } a_{s}^{\dagger}=0
$$

and one may introduce the phase operator of Pegg and Barnett in (7), which is unitary $\mathrm{e}^{i \phi}\left(\mathrm{e}^{i \phi}\right)^{\dagger}=\left(\mathrm{e}^{i \phi}\right)^{\dagger} \mathrm{e}^{i \phi}=1$ in $(s+1)$-dimensional space. The large $s$-limit of this construction leads to the problematic aspects arising from index mismatch analysed in Ref[5]. Also, the large $s$-limit of (33) does not lead to the standard representation (20) with well-defined Casimir operator, since $n_{0}=\frac{s+1}{4}$ in $(\sqrt{33})$.

Another view of the representation (31), which is interesting from an index consideration, is to regard (31) as an infinite dimensional representation specified by the Casimir operator $c=\left[n_{0}\right]$ with $-n_{0}$ the lowest eigenvalue of $N$. We then have the kernels

$$
\begin{aligned}
\text { ker } a & =\{|0>,| s+1>\mid 2 s+2>, \cdots\} \\
\operatorname{ker} a^{\dagger} & =\{|s>,| 2 s+1>, \cdots\}
\end{aligned}
$$

and

$$
\operatorname{dim} \text { ker } a=\infty, \quad \text { dim } \operatorname{ker} a^{\dagger}=\infty
$$

Consequently, (31) corresponds to a singular point of index theory where the notion of index becomes ill-defined: we have no constraint on the phase operator arising from an index consideration. In fact, one may accomodate either the non-unitary $\mathrm{e}^{i \varphi}$ in (4), which is normally associated with

$$
\text { dim ker } a-\operatorname{dim} \text { ker } a^{\dagger}=1 \text {, }
$$

or a unitary $\mathrm{e}^{i \Phi}$ defined by 


$$
\begin{aligned}
\mathrm{e}^{i \Phi}= & |0><1|+|1><2|+|2><3|+\cdots+\mathrm{e}^{i \phi_{0}}|s><0| \\
& +|s+1><s+2|+\cdots+\mathrm{e}^{i \phi_{1}}|2 s+1><s+1| \\
& +\cdots
\end{aligned}
$$

with $\phi_{0}, \phi_{1}, \cdots$, real constants; unitary $\mathrm{e}^{i \Phi}$ is normally associated with

$$
\text { dim ker } a-\operatorname{dim} \text { ker } a^{\dagger}=0 .
$$

Both of these phase operators give rise to the same representation for $a$ in (31),

$$
\begin{aligned}
a & =\mathrm{e}^{i \varphi} \sqrt{[N]+\left[n_{0}\right]} \\
& =\mathrm{e}^{i \Phi} \sqrt{[N]+\left[n_{0}\right]}
\end{aligned}
$$

However, we have no more the expression in (29) since $[N+1]+\left[n_{0}\right]$ can vanish. The operator $\mathrm{e}^{i \Phi}$ gives rise to the same physical implications as $\mathrm{e}^{i \phi}$ in (7) for the physical states defined in (12).

\section{Discussion and Conclusion}

We would like to summarize the implications of the above analysis. First of all, the notion of index is well-defined for a real positive $q$ (which includes $q=1$ ), and the index is invariant under a continuous deformation specified by $q$. The notion of index presents a stringent constraint on the possible form of the phase operator.

For $q=\exp (2 \pi i \theta)$, the notion of index becomes subtle. Since the rational values of $\theta$ are densely distributed among the real values of $\theta$, one cannot define a notion of continuous deformation for the index (i.e., $\operatorname{dim} \operatorname{ker} a$ - $\operatorname{dim} \operatorname{ker} a^{\dagger}$ ); one encounters singular points associated with a rational $\theta$ almost everywhere. Only when one regards the singular situation such as in (36) as corresponding to the index relation 


$$
\operatorname{dim} \text { ker } a-\operatorname{dim} \text { ker } a^{\dagger}=1
$$

one maintains the notion of continuous deformation. Even in this case, there is certain complication for $\theta \rightarrow 0$ to reproduce the normal case of $q=1$ if one sticks to representation (23); the Casimir operator cannot be well-defined in the limit $\theta \rightarrow 0$ as it should be.

If one formally defines the representation 3

$$
\begin{aligned}
a & =\sum_{k=1}^{\infty} \sqrt{[k]}|k-1><k| \\
a^{\dagger} & =\sum_{k=1}^{\infty} \sqrt{[k+1]}|k+1><k| \\
N & =\sum_{k=0}^{\infty} k|k><k| \\
c & =0
\end{aligned}
$$

for all allowed values of $q$ and if one formally takes the index (39) even for a rational $\theta$, one can maintain the notion of continuous deformation of the algebra and its representation. Only in this case, the index as well as the phase operator remain invariant under $q$-deformation. The standard finite dimensional representation for $q=\exp (2 \pi i \theta)$ with a rational $\theta$ may be interpreted that the well-defined notion of index, which is supposed to be invariant under deformation, is lost for a rational $\theta$ and the representation makes a discontinuous transition to finite dimensional irreducible representations.

In conclusion, the notion of index, when it is well-defined, is useful as an invariant characterization of $q$-deformation of an algebra. In addition, we have also shown how to overcome the problem of negative norm for $q=\exp (2 \pi i \theta)$.

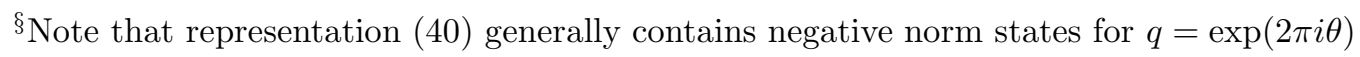




\section{References}

[1] P A M Dirac, Proc. Roy. Soc A114 (1927) 243.

[2] L Susskind and J Glogower, Physics, 1 (1964) 41.

[3] D T Pegg and S M Barnett, Phys Rev A39 (1989) 1665.

[4] P Carruthers and M M Nieto, Rev Mod Phys 40 (1968) 411, and references therein.

[5] K Fujikawa, to appear in Phys. Rev. A; hep-th 9411066.

[6] A J MacFarlane, J Phys A: Math Gen 22 (1989) 4581.

[7] L C Biedenharn, J Phys A: Math Gen 22 (1989) L873.

[8] Hong Yan, J. Phys A:Math Gen 23 (1990) L1150.

[9] C H Oh and K Singh, J Phys A: Math Gen 27 (1994) 5907; hep-th 9407142.

[10] S H Chiu, R W Gray and C Nelson, Phys Lett A 164 (1992) 237.

[11] E G Floratos and T N Tomaras, Phys Lett B 251 (1990) 163.

D. Ellinas, Phys. Rev. A45 (1992) 3358.

H C Fu and M L Ge, Nankai Report NIM-PH 12 (1994)

[12] G Rideau, Lett Math. Phys. 24 (1992) 147 